\title{
Stand-Off Energy Sources for Z-Pinch Implosions
}

D. Ryutov

A. Toor

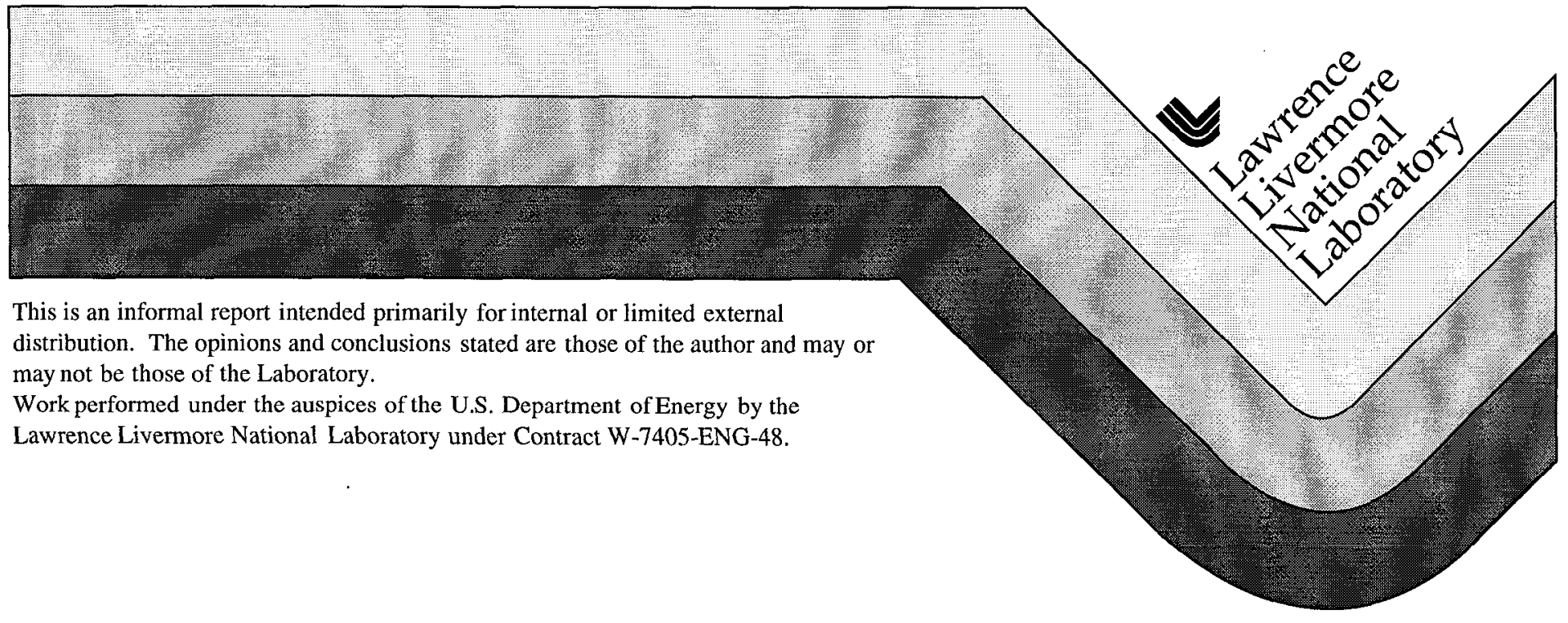




\section{DISCLAIMER}

This document was prepared as an acccount of work sponsored by an agency of the United States Government. Neither the United States Government nor the University of California nor any of their employees, makes any warranty, express or implied, or assumes any legal liability or responsibility for the accuracy, completeness, or usefulness of any information, apparatus, product, or process disclosed, or represents that its use would not infringe privately own rights. Reference herein to any specific commercial products, process, or service by trade name, trademark, manufacturer, or otherwise, does not necessarily constitute or imply its endorsement, recommendation, or favoring by the United States Government or the University of California. The views and opinions of authors expressed herein do not necessarily state or reflect those of the United States Government or the University of California, and shall not be used for advertising or product endorsement purposes.

This report has been reproduced directly from the best available copy.

A vailable to DOE and DOE contractors from the Office of Scientific and Technical Information P.O. Box 62, Oak Ridge, TN 37831

Prices available from (615) 576-8401, FTS 626-8401

Available to the public from the National Technical Information Service

US. Department of Commerce 5285 Port Royal Rd., Springfield, VA 22161 


\title{
Stand-off energy sources for Z-pinch implosions
}

\author{
D. Ryutov, A. Toor
}

(LLNL, Livermore)

\section{Introduction}

In this memo, we discuss several versions of stand-off energy sources for Z-pinch implosions. We concentrate on the sources which are totally mechanically and electrically uncoupled from the "external world." A single exception is a concept discussed in Sec. 4 (where a direct link of the diode with the external energy source is allowed).

A possible way of solving the problem of a stand-off energy source has been delineated in Refs. [1,2], where it was suggested that the fusion reactor would work in the following way: the disposable assemblies (with the size of $30-50 \mathrm{~cm}$ ) would be dropped into reaction chamber (whose walls would be protected by liquid $\mathrm{Li}$ or $\mathrm{LiPb}$ flow, very much like in other ICF reactors, Ref. [3]), and the energy required to drive the implosion would be delivered from the distance of tens of meters (see below). It was assumed that the assembly would contain an energy conversion system, a liner with a fusion target, and onboard circuitry (possibly required for a proper conditioning of the power pulse).

There are several ways of delivering the energy to the assembly dropped into the reaction chamber. The first is based on the use of fast flyers [1,2] accelerated either electromagnetically [4] or explosively [5], or by some other, presently unknown, techniques. These flyers could then be used to compress the conducting flux conserver that encloses some seed magnetic field (the latter could be generated, in particular, by the inverse diode system, see below). The kinetic energy of the flyer would be converted into the energy of the magnetic field and the latter would drive a circuit of the imploding liner. The second is an "inverse diode" system [1,2], where the assembly would be energized by a $1-\mathrm{MeV}$ electron beam, penetrating into the assembly through the entrance foil, being absorbed by the cathode, and generating a voltage between the foil and the cathode. With an appropriate circuitry (including, possibly, a pulse transformer) installed in the assembly, 
this energy source could be used to drive some fast circuits, or to create a seed magnetic field in the flux-conserver in the fasst-flyer scheme. The third approach employs generating supra-thermal electrons by illuminating a kind of a thermoionic diode attached to the "assembly" by intense light of a low-quality (and, accordingly, relatively inexpensive) $\mathrm{CO}_{2}$ laser [6]; these fast electrons could then be used, e.g., to drive a current and generate the seed magnetic field in the flux conserver. A brief discussion of these and some other techniques can be found in Refs. [7, 8]. As was noted in Ref. [9], it is strongly advisable to make the assembly mostly of the same materials as the protective liquid layer flowing along the inner surface of a liner (to avoid additional complications with chemical separation). This, essentially, limits the main materials to lithium and lead (plus, in some cases, to flibe).

An important question for energy applications is that of the cost of each disposable assembly, which should not exceed a few cents per ten megajoules of the fusion energy. This issue is, however, beyond the scope of our study.

In this paper, we provide a more detailed analysis of the scheme based on the use of the fast projectile (Sec. 2). In Sec. 3, we make an attempt to develop the idea of paper [9] (using MHD converter driven by the evaporated material initially surrounding the target) in the direction of smaller fusion yields. In Sec. 4, we consider a possibility of making disposable interfaces of a cold porous lithium, lithium-lead, or flibe, to directly connect the $Z$ pinch diode with external (permanent) energy source.

\section{Stand-off energy source based on the use of a fast projectile}

As a reference case we take the case where the energy delivered to the Z-pinch liner is $5 \mathrm{MJ}$. Assuming that the conversion efficiency from the fast projectile to the liner is $50 \%$, one finds that the initial energy delivered to the assembly should lie in the range of 10 MJ. We will use these numbers as a reference point, although most of the equations will be presented in a general form. 


\subsection{Energies and dimensions.}

We assume that there is a conducting flux-conserver enclosing seed magnetic field with the field strength of the order of $100 \mathrm{kG}$. The initial magnetic energy density $W_{0}$ is then:

$$
W_{0}=40 \mathrm{~J} / \mathrm{cm}^{3}
$$

If the cross-section of the flux conserver is rapidly compressed and becomes by a factor of $K$ smaller than the initial cross-section, the energy stored in the magnetic field increases by a factor of $K$ (whereas the magnetic field density increases by a factor of $K^{2}$, $W=K^{2} W_{0}$ ). If one is interested in delivering to the load an energy $U$, the initial volume $\mathrm{V}_{0}$ containing the seed magnetic filed should be

$$
V_{0}=U /\left(K W_{0}\right)
$$

Taking $U=5 \mathrm{MJ}, K=20, W_{0}=40 \mathrm{~J} / \mathrm{cm}^{3}$, one finds that the initial volume enclosed by the flux conserver should be $V_{0}=6 \ell$. In the scheme shown in Fig. 1, the projectile energy is initially converted into the energy of the compressed magnetic field, and gets switched to the load by an opening switch. This switch could be one- or two-staged, depending on the time-history of the compression process. A current-breaker made of a wire-array of the type discussed in Ref. [10] could be used. The curent wave-form in the load can be tailored by the proper tailoring the shape of the flux-conserver.

\subsection{Requirements to the projectile}

The kinetic energy of the projectile is:

$$
U_{k i n}(\mathrm{~kJ})=0.5 \cdot m(\mathrm{~g}) \cdot[\mathrm{v}(\mathrm{km} / \mathrm{s})]^{2}
$$

To have a projectile with the kinetic energy content of $10 \mathrm{MJ}$ at the velocity of $\mathrm{v}=10 \mathrm{~km} / \mathrm{s}$, one has to take a mass of $200 \mathrm{~g}$. For the projectile made of $\mathrm{Pb}$ or $\mathrm{LiPb}$ eutectic, the volume would be $\sim 20 \mathrm{~cm}^{3}$. 
It might be desirable to create the projectiles with the thickness varying over the surface. Using simultaneously a conserver of the shape shown in Fig. 1, one could provide conditions where the impact would first occur with a thinner part of the projectile. The kinetic energy per unit area would match the energy required to squeeze the initial magnetic field out from this peripheral area. Thicker parts of the flyer would impact the parts of a conserver with already compressed magnetic field. The kinetic energy per unit area of the flyer is $\rho v^{2} h / 2$, where $\rho$ is the density, and $h_{f}$ is the thickness of the flyer plate. The energy required to squeeze the magnetic field out of a certain part of the flux conserver is $\left(B^{2} / 8 \pi\right) h_{\text {cons }}$ (per unit area of the flux conserver). The optimum thickness distribution over the surface of a flyer is roughly determined by equating the two expressions, which yields:

$$
h_{f}=\frac{B^{2}}{4 \pi \rho v^{2}} h_{c o n s}
$$

where $B$ is the instantaneous value of the magnetic field at the time of impact. Creating a "matched" flyer allows one (at least on paper) to reach a conversion efficiency approaching $100 \%$.

The front side of the flux conserver should have a mass small compared to the mass of the flyer (so that the energy losses in the inelastic impact would be minimal), while the opposite side should be thick enough not to yield too strongly to the magnetic pressure (see Sec. 2.5 for a discussion of the latter problem).

After the impact, the flyer (with the front surface of the conserver stuck to it) would experience deceleration by the magnetic pressure and would therefore suffer from the Rayleigh-Taylor instability. Hovever, the deceleration time is only by a factor of a few longer than the e-folding time for the most dangerous perturbations with the wavelength of order of the flyer thickness. Therefore, this instability should not cause particularly severe problems. 


\subsection{Generation of fast projectiles}

The planar flyers (thin slabs) with velocities of order of $10^{7} \mathrm{~cm} / \mathrm{s}$ have been obtained in electromagnetic accelerators, with the flyer energy $100 \mathrm{~kJ}$ [4]. Explosively driven cumulative jets with velocities up to $9 \cdot 10^{6} \mathrm{~cm} / \mathrm{s}$ were also obtained [5]. Note that the flyer does not need to be in a solid state. One can use liquid jets formed explosively (Fig. 2). Extrapolation to a few megajoules looks feasible, especially with explosively driven flyers. With a perpendicular size of the flux conserver $h_{\text {cons }} 2 \mathrm{~cm}$ (Fig.1), one finds that a characteristic rise-time of the current generated by this magneto-compressive generator is $\sim 2 \mu \mathrm{s}$, matching the natural time-scale of the problem.

\subsection{Resistive losses}

At the level of the magnetic field $\sim 2 \mathrm{MG}$ which we expect to be reached in the flux -conserver by the end of the compression process, the inner surface of the flux conserver will be molten but not yet vaporized. It is therefore reasonable to use a resistivity of molten metals as a reference number in the cstimates of the resistive losses. Taking, as a representative value, the resistivity of the liquid lead at $600^{\circ} \mathrm{C}, \eta=60 \mu \mathrm{Ohm} \cdot \mathrm{cm}$, one finds that the magnetic diffusivity is $4.5 \cdot 10^{3} \mathrm{~cm}^{2} / \mathrm{s}$. For a pulse-width of $t=2 \mu \mathrm{s}$, this yields the skin-depth of $1.5 \mathrm{~mm}$. This should be compared to the characteristic size of the compressed flux-conserver, which is a few centimeters (the volume $\sim 300 \mathrm{~cm}^{3}$ ). The skin thickness divided by the linear dimension of the conserver gives the value of the Ohmic energy losses, which is less than $10 \%$.

\subsection{Other issues of the assembly design.}

The imploding liner and the target should not be shaken mechanically before and in the course of the implosion process. This basically means that the current pulse should 
arrive at the target before the acoustic perturbations do. This condition will be satisfied if the compression of the flux conserver occurs with the velocity exceeding the sound speed in the leads. If necessary, one can somewhat increase the length of the electric leads connecting the diode with the flux-conserver.

The leads would experience a magnetic pressure and would yield to it in the normal direction. To reduce this effect to a permissible level, one could make the leads of a heavy material, say, lead, even if the rest of the assembly is made of the lighter material, say, lithium. The magnetic pressure is related to the magnetic field strength by the equation

$$
p_{M}(k b a r)=40[B(M g)]^{2}
$$

A normal displacement $\delta$ of a planar conductor of a thickness $h$ within the time $t$ can be evaluated as:

$$
\delta(m m) \approx 5 \cdot 10^{2} \frac{p_{M}(k h a r) t^{2}(\mu s)}{\rho\left(g / \mathrm{cm}^{3}\right) h(m m)}
$$

where $\rho$ is the mass density of the conductor. Assuming that the magnetic field in the planar transmission line is $2 \mathrm{MG}$, that the conductor is made of lead with a thickness 2 $\mathrm{mm}$, and that the pulse-width is $2 \mu \mathrm{s}$, one finds that the displacement is of the order of 1.5 $\mathrm{mm}$, which looks tolerable.

\section{MHD conversion at low yields?}

In his important paper [11], B.G. Logan has analyzed the possibility of using direct MHD conversion in the ICF fusion reactors. The energy-releasing target was supposed to be surrounded by a sphere made of a tritium-breeding material (e.g., lithium). After fusion energy release, this sphere would have been evaporated and ionized (mostly by a volumetric neutron heating), to produce a working fluid for direct energy conversion (and, at the same time, breed tritium). To provide conditions where a significant (say, 95\%) 
fraction of the energy released would be absorbed in this sphere, its radius had to be in the range of $1 \mathrm{~m}$. This size determined the minimum energy yield for which this concept would work (i.e., for which even the outermost layers of the ball would be evaporated and heated to $10^{4} \mathrm{~K}$, making them highly conductive), and which turned to be in the range of several gigajoules to tens of gigajoules. The paper $[11]$ contains a wealth of ideas and detailed calculations pertinent to this approach.

A variation to this approach that we are going to suggest is related to a possibility of a hydrodynamic channeling of the gas formed around the target, and ejecting it from the reactor chamber through a hole that would be opened and closed in a synchronized fashion. Most importantly, we are going to exploit the fact that $20 \%$ of fusion energy is released in the form of alpha-particles, whose range in the surrounding matter is infinitesimally small compared to all other dimensions (unlike for fusion neutrons, for which the range is typically tens of centimeters). We suggest that the alpha energy be used to create an ionized and highly conducting layer that would be pushed from the back by denser and colder poorly conducting gas formed by evaporation of the outer layers of material initially surrounding the target (see for the specifics below). This gas does not now need to be ionized, thereby leading to reduced requirements to the energy yield. We contemplate of using a pulsed inductivcly-coupled version of the the MHD converter of the type shown in Fig. 3. $\Lambda$ different version of the pulsed MHD converter, as well as a summary of the earlier proposals of this type, can be found in Ref. [12].

To produce a hot ionized gas, we suggest to make the inner layer of the sphere, nearest to the target, of a low-density foam. This foam layer will be $-10 \mathrm{~cm}$ thick. $\Lambda \mathrm{s}$ the fusion energy release in a plasma target will take less than a few nanoseconds, a strong shock will be excited in the foam that will heat and ionize it. The shock will be driven essentially entirely by the energy released in the form of alpha particles. The desired temperature can be controlled by the density of the foam. The thus formed hot conducting gas would be allowed to leave the central part of the ball through a nozzle that would direct 
it to the entrance aperture of the MHD converter. Because of a high expansion speed of this hot gas, it will arrive at the entrance hole of the converter earlier than the gas from the outer layers vaporized by the neutron irradiation.

This colder and poorly conducting gas will also be directed to the MHD generator by properly distributed heavy material limiters (made, e.g., of $\mathrm{LiPb}$ eutectic). 'These latter will also be vaporized (not to produce shrapnel) but will expand much slower than the lighter jet. Their energy would have to be removed by a standard steam cycle (or just dumped into the secondary cooling contour). The (potential) advantage of this scheme is in that it will need only to vaporize (not to ionize) the main part of the sphere, thereby reducing requirements to the fusion yield per shot by a factor of a few.

To further reduce the yield compatible with the concept of the evaporated ball, one could reduce its radius from more than a meter to a few tens of centimeters, sacrifying part of the neutron energy release. According to calculations of the neutron energy deposition in various candidate materials (flibe, $\mathrm{Li}$, and $\mathrm{LiPb}$ ) presented in Ref. [11] (with the reference to analysis by Sahin), for a fusion energy yield of 200-300 MJ one can expect approximatcly $50 \%$ of the yield would be converted into the the internal energy of the vapor. This part of the cncrgy would then be converted to electricity with a high efficiency determined by a high conductivity of the lcading part of the jet.

The rest of the energy would be deposited to a liquid protcctivc/brceding layer on the inner side of the reaction chamber. The actual desirability of converting this part of the energy into electricity by a lower-efficiency steam cycle, is a matter of a more detailed economic analysis of the electric plant.

If the energy is supplied to the ball and a pinch nested inside it by a fast flyer, the crushed flux-conserver can be situated outside the ball, with the power transmitted to the pinch through a narrow slot (power transmission line). Alternatively, the flyer made of a heavy material could be integrated with the outer part of the ball after the impact. Designing 
such integrated systems could be an interesting although challenging hydrodynamic problem.

These are preliminary consideration not supported yet by more detailed analysis, which may reveal both hidden difficulties and hidden opportunities. In particular, it could be interesting to explore pros and cons of using lithium hydride as a material for the neutron-absorbing sphere [11]. Because of its neutronic properties, even a relatively small sphere of $\mathrm{LiH}(\sim 15 \mathrm{~cm}$ in diameter) would absorb nearly $90 \%$ of the neutron energy [11]. This property is very important if one is concerned with low-yield systems: amount of energy required to vaporize this small sphere is in the range of 200-300 MJ. Hydrodynamic channeling of the gas into MHD converter would allow one to use this gas at high pressure, without letting it to expand over the whole volume of the reaction chamber.

\section{Using porous materials for disposable interface between the target and external generator.}

In this section we discuss a possibility of using disposable inserts that would provide direct electrical contact of the diode with the external power source. This approach could be preferential for those versions of fusion reactors where the energy delivered to the target is supposed to be in the range of $20-30 \mathrm{MJ}$ or more (it would probably be difficult to deliver such amounts of energy by totally detached energy sources of the type discussed in

Secs. 1 and 2). Following suggestion made in Ref. [8], we discuss here potential advantages of making disposable insert of porous $\mathrm{Li}$ or $\mathrm{LiPb}$. General schematic of a disposable insert is shown in Fig. 4. The whole insert should be used at a low temperature in the range of $-20 \mathrm{C}$ in order to make the porous material stiff enough. The insert will be combined with a canonical "waterfall" protection of the reactor chamber (Fig. 4).

The use of porous materials provides the following potential advantages:

1. The mass of the insert can be made much smaller than for solid materials, thereby facilitating a quick replacement of the insert. 
2. Using materials with varying porosity, one could exploit effect of hydrodynamic lensing [13] in order to direct evaporated materials away from the slots in the reaction chamber through which the power flows in from the external generator.

3. By making the mass of the whole disposable part small enough, one can rely on its evaporation by the shock heating (Cf. Sec. 3), thereby eliminating the problem of shrapnel.

The total line-density of the porous material along the shortest line of sight to the wall surface should be sufficient to intercept, say, $95 \%$ of the neutrons. This may require using a solid (not porous) blanket at the bottom of the insert. This solid part might be inserted at the temperature close to the melting temperature before the fusion pulse, and get molten after the pulse.

\section{Summary}

An issue of stand-off energy sources is in an early stage of development. Several concepts have been identified as potential solution of the problem. Those based on the total disconnection between the target assembly and the primary energy source have an obvious advantage in that they allow one to relatively easily protect the permanent part. Indeed, a fast projectile travelling at the velocity of $10 \mathrm{~km} / \mathrm{s}$ covers the distance of $10 \mathrm{~m}$ in $1 \mathrm{~ms}$, the time that is sufficient to mechanically shield the line of site. Auxiliary power supply in the form of an electron beam can be protected by using a magnetic wiggling in the transport channel in the permanent part of the facility. Of some help is also the fact that this auxiliary source operates 10 or so microseconds before the fusion energy release occurs. Another advantage of this approach is related to its compatibility with high rep-rate mode, up to tens pulses per second (because there is no need to insert heavy large-volume parts into the reaction chamber). An obvious disadvantage is that the assembly should contain a more or less complex on-board circuitry. 
Those concepts that are based on the direct mechanical connection between the external pulse-forming line and the disposable inner part of the transmission line (connected in turn to the Z-pinch diode) have an obvious advantage in eliminating any intermediate steps in delivering the energy to the pinch. They work essentially as the presently used Zpinch devices and should provide the same high efficiency. A difficulty of this approach is related to the presence of the insulating slot in the walls of the reaction chamber that directly links the disposable inner part of the energy transmission system and permanent external energy source. The slot is vulnerable to mechanical perturbations and neutron irradiation that may propagate into the permanent part of the source and cause a damage to it. Mechanical damage could be reduced by the technique of hydrodynamic channeling or hydrodynamic lensing mentioned in Secs. 3 and 4.

Those concept that are based on the total disconnection between the target assembly and the primary energy source have an obvious advantage in that they allow one to relatively easily protect the permanent part. Indeed, a fast projectile travelling at the velocity of $10 \mathrm{~km} / \mathrm{s}$ covers the distance of $10 \mathrm{~m}$ in $1 \mathrm{~ms}$, the time that is sufficient to mechanically shield the line of site. Auxiliary power supply in the form of an electron beam can be protccted by using a magnetic wiggling in the transport channel in the permanent part of the facility. Of some help is also the fact that this auxiliary source operates 10 or so microseconds before the fusion energy release occurs. Another advantage of this approach is related to its compatibility with high rep-rate mode, up to tens pulses per second (because there is no need to insert heavy large-volume parts into the reaction chamber). An obvious disadvantage is that the assembly should contain a more or less complex on-board circuitry.

We haven't touched upon any economics issues. Some guidance in these matters can be obtained from Refs. [11, 14] related to pulsed high-yield versions of fusion reactors. 


\section{Acknowledgment}

This work was carried out under the auspices of the U.S. Department of Energy by Lawrence Livermore National Laboratory and Sandia National Laboratories under contracts W-7405-ENG-48 and DE-AC04-94AL85000. 


\section{References}

1. Drake, R.P., J.H. Hammer, C.W. Hartman, L.J. Perkins, D.D. Ryutov. "Adiahatic compression of a closed-field-line configuration by a centimeter-size liner". Proc. 16th Symposium on Fusion Engineering, Sept. 30-Oct. 5, 1995, v. 1, p. 97.

2. Drake, R.P., J.H. Hammer, C.W. Hartman, L.J. Perkins, D.D. Ryutov. "Submegajoule liner implosion of a closed field line configuration." Fusion Technology, 30, 310, 1996.

3. Moir, R. Phys. Plasmas, 2, 2447 (1995).

4. I.E. Osher, J.F., G. Barnes, H.H. Chau, R.S. Lee, C. Lee, R. Speer, and R.C. Weingart. IEEE Trans. Plasma Sci., 17, 392 (1989)

5. Lavrent'ev, M.A., and B.V. Shabat. "Hydrodynamical Problems and their Mathematical Models," Moscow, Nauka Publishing House, 1973 (in Russian)

6. Ryutov, D.D. "Stand-off energy sources for pulsed fusion devices." Innovative Conf. Concepts Workshop, PPPL, April 4-6 1998, UCRL-JC-129697 Abs.

7. Ryutov, D.D. "A user facility for research on fusion systems with dense plasmas" Proc. of the 2nd Symp. "Current Trends in International Fusion Research," Wash. DC, March 1997, To be published by NRC Research Press, Ottawa, Canada. LLNL Report UCRL-JC-126188.

8. Ryutov, D.D. "A problem of stand-off energy sources for MTF," LLNL report UCRLJC-133716, April 19, 1999.

9. Ryutov, D., Toor, A. Unpublished.

10. Logan, B.G., and J.H. Hammer. Private communication, 1998

11. Logan, B.G. "Inertial fusion reactors using Compact Fusion Advanced Rankine (CFARII) MHD conversion," Fusion Engineering and Design, 22, 1953 (1993). 
12. Lasche, G.P. "The feasibility of a laser or charged-particle-beam fusion reactor concept with direct electric generation by magnetic flux compression," UCRL 53434, LLNL, 1983.

13. Whitham, G.B. "Linear and Nonlinear Waves." NY, Wiley, 1974.

14. Moses, R.W., R.A. Krakowski, R.L. Miller. "A conceptual design of the fast-liner reactor (FLR) for fusion power." Report LA-7686-MS, February 1979 (http://libwww.lanl.gov/la-pubs/00209074.pd() 


\section{Figure captions}

Fig. 1 A concept of an energy source based on the fast flat flyer plate: 1-slots through which the flux-conserver is initially energized by an auxiliary energy source; these slots get sealed by the flyer early in time; 2-conducting walls of the flux conserver; 3 - the volume occupied by the magnetic field; 4-current breaker of the type considered in Ref. 10; 5empty volumes needed to ensure a complete current breaking; 6-final segments of the leads; 7-Z pinch. Shaded is a flyer of a varying thickness approaching the assembly from the left. Dash-dotted line is the axis of symmetry.

Fig. 2 Using a high-speed liquid metal jet in the magneto-compressive generator. The annular jet (dotted) is approaching the flux-conserver from the left. Its shape is chosen in such a way that the impact on the flux-conserver first occurs in its outermost zone, sealing the slots needed to generate initial magnetic flux. Shaded is a heavy cone protecting the target zone. The other elements are the same as in Fig. 1.

Fig. 3 A concept of a pulsed MHD converter. The target is situated in the central small circle, surrounded by a porous material (dotted zone). Outside this zone, there is a solid sphere, enclosed in turn into a vessel made of a heavier material, with another nozzle. All this material (including the outermost layer) is evaporated in each shot. The hot ionized jet is formed in the annular inner nozzle and is ejected to an MHD channel ahead of the heavier denser gas. MHD channel is not to scale. Arrows show the direction of expansion of the conducting layer under the action of the pressure of a dense neutral gas.

Fig. 4 A porous insert providing a direct connection between the external energy source and $Z$ pinch. 
$06 / 30 / \theta \theta \quad 13: 25$ FAX $925 \quad 423 \quad 3484$

MFE / Theory

幽002

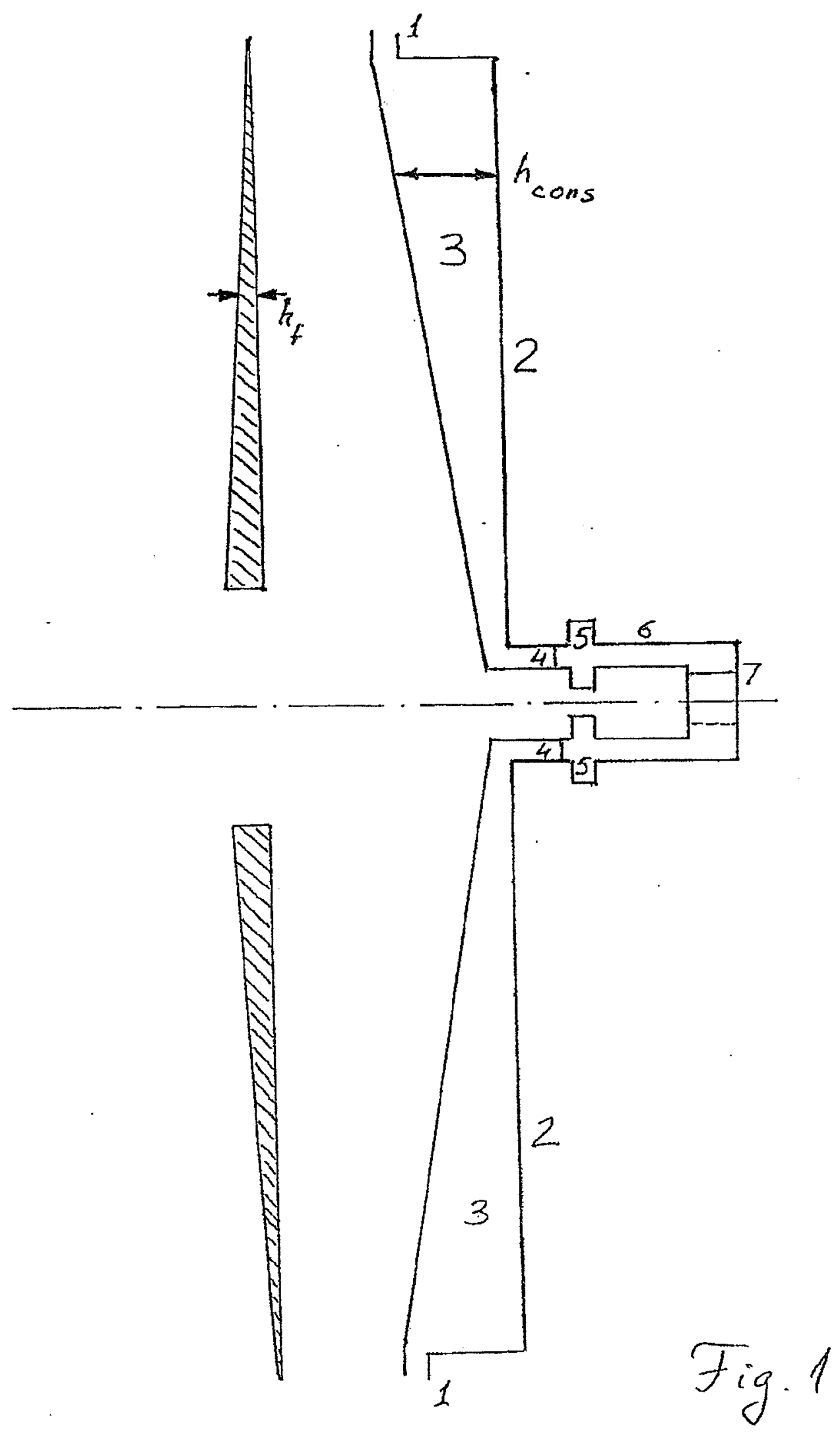




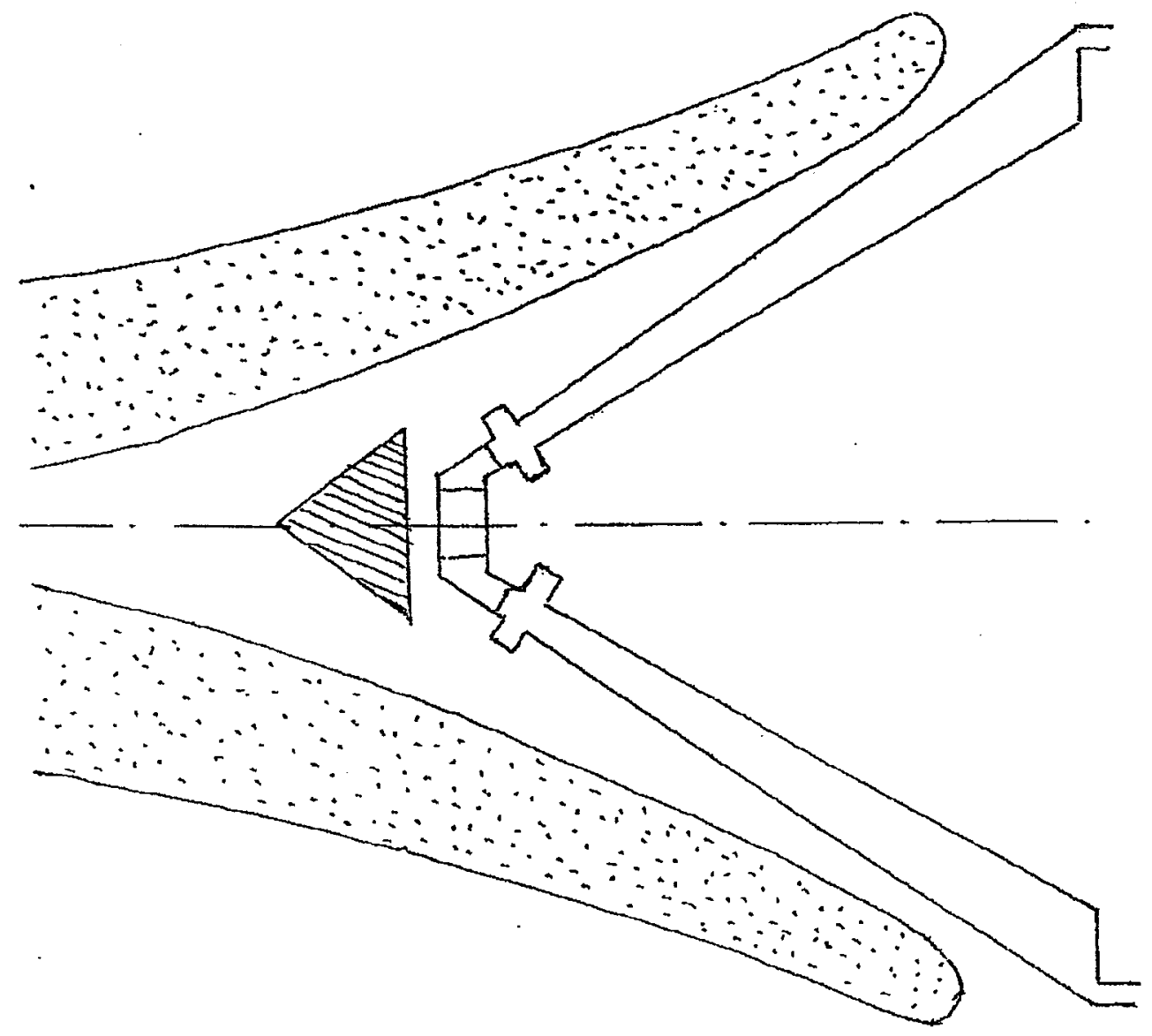

Fig. 2 


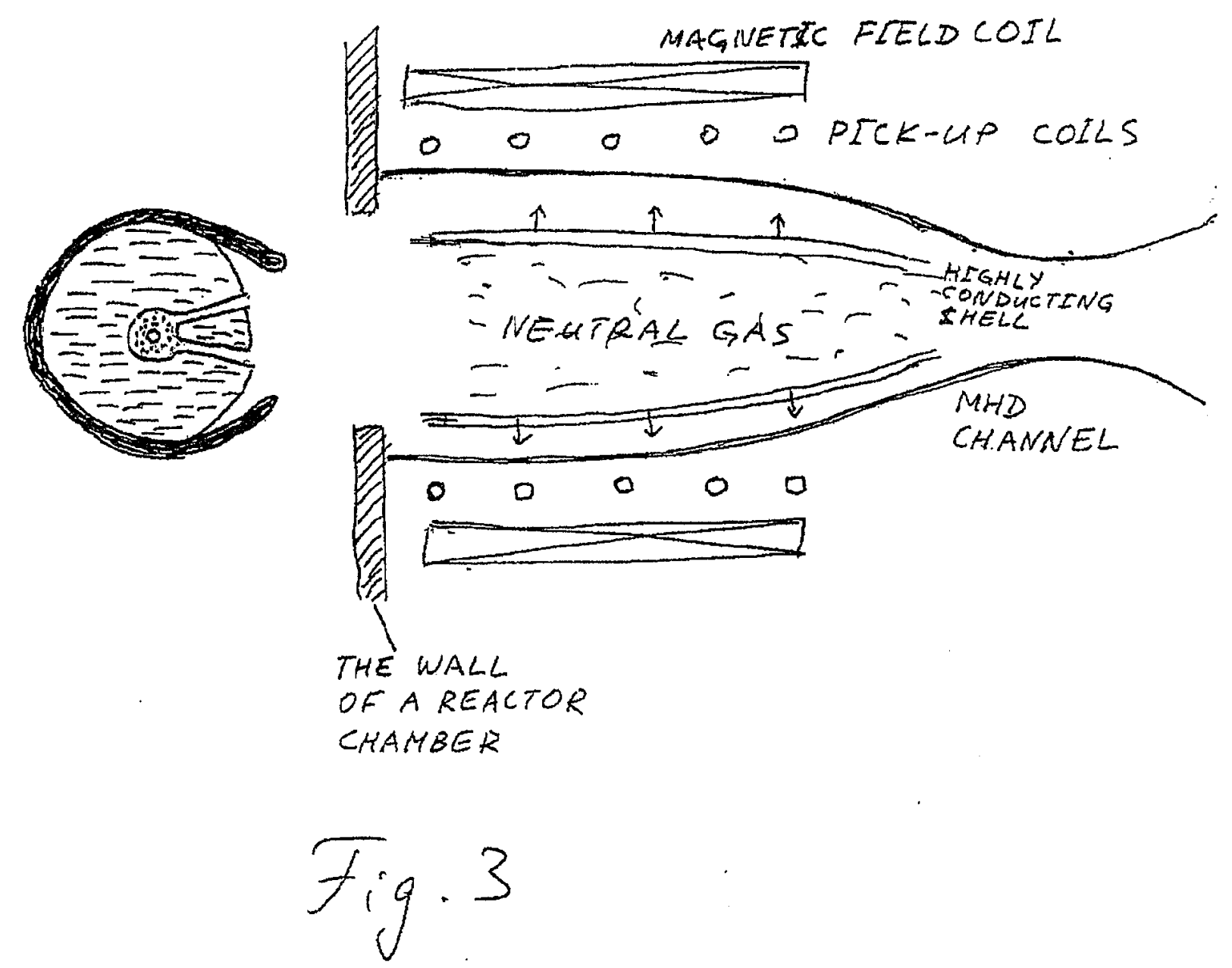


A SOLID INSERT FOR HIGHER-YIELD (>200 MJ) SYSTEMS
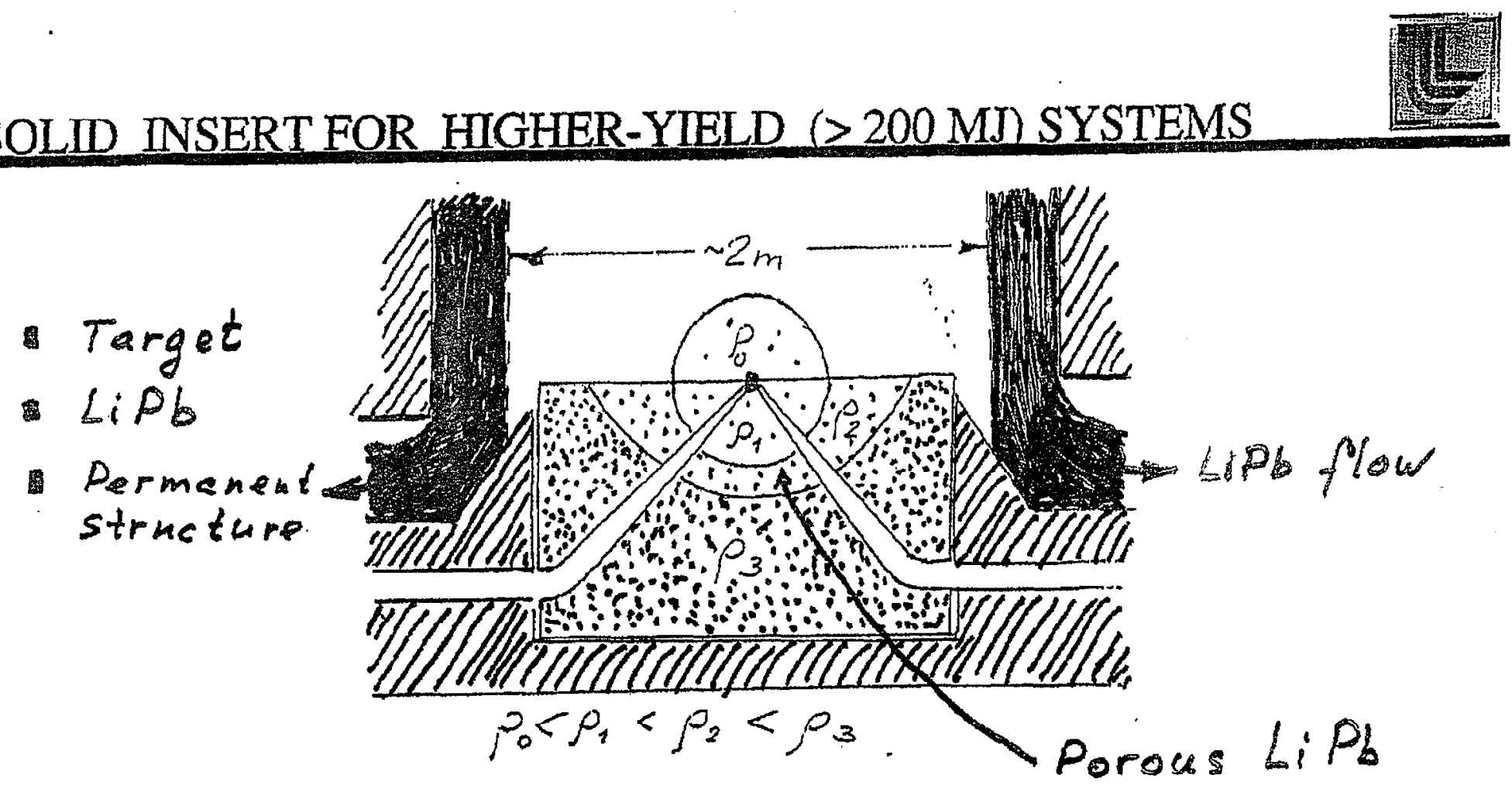

- The material: a porous $\mathrm{LiPb}$ (or $\mathrm{Li}$, or $\mathrm{Pb}$ ) at $\mathrm{T}<-20 \mathrm{C}$

- Density distribution in the vicinity the target chosen in such a way as to exploit the effect of hydrodynamical lensing and direct the ejected material away from the most vulnerable elements of the reaction chamber

- Replacement rate $\sim 1 \mathrm{~Hz}$

$$
\text { Fig. } 4
$$

